\title{
Students' metarepresentational competence with matrix notation and Dirac notation in quantum mechanics
}

\author{
Megan Wawro®, ${ }^{1, *}$ Kevin Watson, ${ }^{1, \dagger}$ and Warren Christensen ${ }^{2}$ \\ ${ }^{1}$ Department of Mathematics, Virginia Tech, Blacksburg, Virginia 24060, USA \\ ${ }^{2}$ Department of Physics, North Dakota State University, Fargo, North Dakota 58108, USA
}

(Received 17 March 2020; accepted 24 July 2020; published 25 August 2020)

\begin{abstract}
This article shares analysis regarding quantum mechanics students' metarepresentational competence (MRC) that is expressed as they engaged in solving an expectation value problem, which involves linear algebra concepts. The particular characteristic of MRC that is the focus of this analysis is students' critiquing and comparing the adequacy of representations, specifically matrix notation and Dirac notation, and judging their suitability for various tasks. With data of students' work during semistructured individual interviews, components of students' MRC were analyzed and categorized according to value-based preferences, problem-based preferences, and purpose and utility awareness. Detail is provided on two students who serve as paradigmatic examples of students' power and flexibility within different notation systems, and detail of a third student is given as a point of contrast. In addition to adapting MRC as a helpful construct for characterizing student understanding at the intersection of undergraduate mathematics and physics, we aim to demonstrate how students' rich understanding of linear algebra and quantum mechanics includes and is aided by their understanding and flexible use of different notational systems. For example, the problem-based preference aspects of MRC highlight that any particular problem-solving approach is itself intrinsically tied to a notational system. We suggest that any instruction with the goal of helping students develop a deep understanding of quantum mechanics and linear algebra should provide opportunities for students to use and improve their MRC.
\end{abstract}

DOI: 10.1103/PhysRevPhysEducRes.16.020112

\section{INTRODUCTION}

As part of a broader study of student reasoning on mathematics and specifically linear algebra within the context of quantum mechanics, we investigate students' metarepresentational competence with matrix and Dirac notation. This study answers the call by the National Research Council's report [1], which charges the U.S. to improve its undergraduate science, technology, engineering, and mathematics (STEM) education, specifically identifies that "gaps remain in the understanding of student learning in upper division courses" (p. 199). Our broader study contributes to this national need by investigating students' understanding, symbolization, and interpretation of eigentheory and related key ideas from linear algebra in quantum physics courses. The particular research question that we report on in this paper is: What aspects of metarepresentational competence exist in students' reflections on and

\footnotetext{
*mwawro@vt.edu

Deceased.
}

Published by the American Physical Society under the terms of the Creative Commons Attribution 4.0 International license. Further distribution of this work must maintain attribution to the author(s) and the published article's title, journal citation, and DOI. comparisons of matrix notation and Dirac notation in quantum mechanics?

This research question arose for us as we examined data from individual interviews that we conducted with students at the end of a quantum spins course. One student's work in solving quantum mechanics problems was particularly striking to us because of the ease with which he moved between and explicitly discussed different notations, namely, Dirac notation and matrix notation. Note his response to the question, "So how do you feel like using eigenvectors and eigenvalues in Spins has been similar to and different from how you've experienced those in other classes?" which was asked at the start of his interview:

Buzz ${ }^{l}$ : Uh, well, it's very similar because you're doing a lot of the same math...the difference, especially in physics, you're looking at kets. In, in at first it was kind of jarring, like to- to try to do the math in kets. But now, it's kind of- it's kind of easier, there's problems, there are certain problems... where there's two ways to do them, they're kind of parallel, you can do it and you can expand the- the- the state in- in like as a- and expand them as- as kets in a different basis, or you can write that

\footnotetext{
${ }^{1}$ All student names are pseudonyms.
} 
TABLE I. Two definitions of linear transformation.

Linear Algebra (Friedberg, Insel, \& Spence, 2003, p. 65)

Let $V$ and $W$ be vector spaces (over $F$ ). We call a function

$T: V \rightarrow W$ a linear transformation from $V$ to $W$ if, for all

$x, y \in V$ and $c \in F$, we have:

(a) $T(x+y)=T(x)+T(y)$ and

(b) $T(c x)=c T(x)$.
Introduction to Quantum Mechanics (Griffiths, 2005, p. 441)

A linear transformation $\hat{T}$ takes each vector in a vector space and "transforms" it into some other vector $\left(|\alpha\rangle \rightarrow\left|\alpha^{\prime}\right\rangle=\hat{T}|\alpha\rangle\right)$, subject to the condition that the operation be linear: $\hat{T}(a|\alpha\rangle+$ $b|\beta\rangle)=a(\hat{T}|\alpha\rangle)+b(\hat{T}|\beta\rangle)$ for any vectors $|\alpha\rangle,|\beta\rangle$ and any scalars $a, b$. state as a- as a, as a vector, in that basis, and you can either do the matrix math for the like expectation values for example, you can do the matrix math or you can do the ket math, and sometimes it's, I'm finding that I'd rather expand something in the ket.

We were immediately grabbed by his awareness that he had a choice in which notation to use and that he had grown in his own facility with the new (to him) Dirac notation. This led us to review research literature on students' understanding of symbols, notations, and representations; we decided the framework of metarepresentational competence [2] aligned with our research goals and provided a powerful construct with which to explore this phenomenon in our dataset.

In this article, we explore the notion of metarepresentational competence (MRC) at the intersection of quantum physics and linear algebra by sharing a detailed analysis of three students' reflections on explicit symbolization choices they made while solving an expectation value problem. ${ }^{2}$ We focus on their reasons for how and why they chose a specific symbol system-either Dirac notation or matrix notation-for that particular situation. We also analyze a third student, who seemed less successful with the interview task at first, and highlight their reasoning as a point of contrast. In addition to offering MRC as a helpful construct for characterizing student understanding at the intersection of undergraduate mathematics and physics, we aim to demonstrate how students' rich understanding of linear algebra and quantum mechanics includes and is aided by their understanding and flexible use of different notational systems.

\section{BACKGROUND, THEORETICAL FRAMING, AND LITERATURE}

Students who are physics majors are exposed to a variety of mathematical concepts and symbols throughout their undergraduate courses. When they begin studying quantum mechanics, they are introduced to a new notation system (Dirac notation) and are expected to align it with previouslytaught concepts and notation systems. For example, consider the two definitions of "linear transformation" given in

\footnotetext{
${ }^{2}$ We presented an earlier analysis of a subset of these data in Ref. [3].
}

Table I. The one on the left is from a textbook commonly used in linear algebra courses taught in mathematics departments, and the one on the right is from a textbook commonly used in quantum mechanics courses taught in physics departments. By the time students are enrolled in the quantum mechanics course, the concept of linear transformation should be familiar to them, yet notice the differences in notation used in the two definitions.

Particularly important to quantum mechanics is eigentheory. More specifically, a Hilbert space ( $\operatorname{such}$ as $\mathbb{C}^{2}$ ) can be assigned to a physical system, to every possible state of the physical system is associated a vector in the Hilbert space, and to every possible observable is associated a Hermitian operator (usually given in its matrix form). The only possible result of a measurement is an eigenvalue of the operator, and after the measurement the system will be found in the corresponding eigenstate. A vector representing a possible state is symbolized with a ket, which can behave mathematically like a column vector, such as $|\psi\rangle \doteq\left[\begin{array}{l}a_{1} \\ a_{2}\end{array}\right], a_{1}, a_{2} \in \mathbb{C}$, and is most often normalized due to the probabilistic nature of quantum systems. The complex conjugate transpose of a ket is called a bra, which can behave mathematically like a row vector, such as $\langle\psi| \doteq\left[a_{1}^{*} a_{2}^{*}\right]$. As a particular example, the eigenvalue equations for $S_{x}$ (the operator corresponding to the observable of the $x$ component of intrinsic angular momentum) of a spin-1/2 particle are $S_{x}| \pm\rangle_{x}= \pm \hbar / 2| \pm\rangle_{x}$, where $|+\rangle_{x}$ and $|-\rangle_{x}$ are orthonormal eigenvectors of $S_{x}$ and the corresponding eigenvalues $\pm \hbar / 2$ of $S_{x}$ are the two possible measurement results of the observable. Note that the orthonormality of the eigenstates of the Hermitian operators associated with observables means the relevant inner products of elements of the eigenbasis with each other yield either 1 or 0 (e.g., $\langle \pm \mid \pm\rangle=1$ and $\langle \pm \mid \mp\rangle=0$ for the eigenstates of $S_{z}$ in a spin-1/2 system). When symbolized in terms of this eigenbasis, the matrix representation of $S_{x}$ is

$$
\left[\begin{array}{cc}
\frac{\hbar}{2} & 0 \\
0 & -\frac{\hbar}{2}
\end{array}\right]
$$

Finally, an understanding of these eigenvalue equations and inner products is important for computing the expectation value of observable $A$ for state $\psi$ when utilizing Dirac notation. These calculations require the bra and ket expansion to be expressed in the same eigenbasis as the 
matrix representation of $A$. As one may alternatively choose to calculate the expectation value of an observable using matrix notation, expectation value problems present a rich setting for investigating students' symbolizing and use of eigentheory and change of basis in both Dirac and matrix notation. We further explain why we focus on students solving an expectation value problem in Sec. III.

\section{A. Theoretical framing}

In a project investigating what it means to understand representation and what higher-level skills may be involved in representational competence, Ref. [2] developed the notion of metarepresentational competence (MRC), which they defined as "the faculty to generate, critique, and refine representational forms." They further elaborated that they chose the prefix "meta" to "emphasize that no specific representational skills are implicated...[T]he skills we attend to are broadly applicable, more flexible, and fluid" (p. 118); In Ref. [4], diSessa re-emphasized that the term is not meant to summon the idea of metacognition but rather "purviews that transcend the mere production and use of representations" (p. 294). MRC includes inventing and designing new representations, judging and comparing the quality of representations, understanding the general and specific functions of representations, and learning to use and understand new representations [5]. Put briefly, Ref. [6] stated, "By MRC, we mean the set of critical abilities and understandings about the "how's and why's" of representations" (p. 446).

In this study, we align ourselves with the theory that representations are a sense-making tool [7], in that "the construction of representations on paper during problem solving mediates and organizes one's understanding of mathematical concepts" (p. 101). Furthermore, "In the process of their emergence, the very contents of a representation will afford certain goals to emerge while weakening the emergence of others" (p. 101). This is compatible with our use of MRC, specific to Dirac notation and matrix notation, to investigate student reflections on their own notational preferences in quantum mechanics and how this competence is an important aspect of a sophisticated understanding of linear algebra and quantum mechanical concepts. In analyzing our data, we focus on the aspects of students' MRC that enable them to "critique and compare the adequacy of representations and judge their suitability for various tasks," and "understand the purposes of representations generally and in particular contexts and understand how representations do the work they do for us" (Ref. [4], p. 293).

It is important to note that early papers on MRC $[2,5,8]$ focused mainly on students' abilities to invent and critique representations of physical situations (e.g., inventing drawings or graphs to represent motion; creating programs to make a "spaceship" on a computer move in certain ways [8]). However, there have been a few studies on students'
MRC regarding algebraic representations as well. For instance, Ref. [9] examined MRC in an 8th grade Algebra I classroom where students had to make decisions about representing and solving word problems algebraically. The criteria used by the teacher and students related to the production and critique of equations included aspects such as 'equations should only contain one variable,' 'terms in an equation should be expressed using the same units as the final requested quantity,' and 'an equation should be balanced.' In a related study, Ref. [10] presented a theoretical frame for coordinating how students use, develop, and evaluate representations and the relationship to the reorganization of students' knowledge structures.

Many physics education research studies focus on the importance of students' understanding and use of multiple representations in physics at the university level [11-14]. Similarly, there exists research that investigates students' "representational competence" or "representational fluency" and the assessment of those skills [15-18]. However, there seems to be a dearth of physics education research studies at the undergraduate level that explicitly employ the MRC framework, although compatible findings do exist [e.g., Ref. [19] concludes that "students need to develop an understanding of the disciplinary affordances of different physics representations" (p. 665), which we interpret as a call out for the development of students' MRC]. Thus, in this paper we explore a new direction into students' MRC, namely, students' thoughtful reflection on and use of canonical representations in undergraduate physics. Furthermore, we explore how students' understanding and use of these representations might benefit from their MRC and vice versa. In doing so, we hope this work contributes to diSessa and Sherin's goal of working to delimit the extent and content of MRC [5], specifically with respect to quantum physics students' reflection and judgment regarding Dirac notation and matrix notations.

\section{B. Literature on student understanding of symbols and representations}

Research on student understanding and use of symbols exists throughout both mathematics and physics education research in the $\mathrm{K}-16$ levels. One construct from mathematics education research that is most relevant to our work is Arcavi's notion of "symbol sense" [20-21]. Through working with beginning algebra students, he theorized a collection of behaviors that collectively define symbol sense, including (a) "friendliness" with symbols, (b) a sense of the different roles symbols can play across various contexts, (c) the recognition of symbols' meaning at any step in the solution process; and an awareness of one's ability (d) to engineer symbolic expressions, (e) choose which symbolic representations to employ in a problem setting, and (f) decide which aspects of a mathematical situation to symbolize. We see this as particularly relevant to and compatible with our investigation of students' MRC. 
Specifically, we see behaviors (b), (d), and (e) particularly salient to the specific aspects of MRC we focus on in this paper; namely, how students critique and compare the adequacy and suitability of representations for various tasks, and how students reflect on representations' general and particular purposes.

Research in undergraduate mathematics education has also studied the importance of understanding symbolization in mathematics; for instance, Harel and Sowder [22] describe how mathematical notations play a key role in forming conceptual entities in higher mathematics. The authors describe how higher mathematics makes frequent use of conceptual entities or cognitive objects to aid in problem solving and the "vertical" growth of mathematical knowledge, and that mathematical notations play a key role in forming conceptual entities, aiding in the entification process. Examining students' structure sense in high school and university algebra courses, Novotná and Hoch [23] found reasonable evidence that high school algebra structure sense, which resides mostly in the symbolic world described by Tall [24], is a prerequisite for the formal world structure sense needed in university abstract algebra courses.

In linear algebra research, Hillel [25] offered three modes of description (abstract, algebraic, and geometric) for the basic objects and operations in linear algebra and pointed out that "the ability to understand how vectors and transformation in one mode are differently represented, either within the same mode, or across modes is essential in coping with linear algebra" (p. 199). Wawro [26] investigated the ways in which one student reasoned about solutions to the matrix equations $A x=0$ and $A x=b$ to make and justify logical connections between a variety of concepts in linear algebra. For instance, while trying to explain why "the columns of $A$ span $\mathbb{R}^{3}$ " implies "Any vector $b$ in $\mathbb{R}^{3}$ can be written as a linear combination of the columns of $A$ " for a $3 \times 3$ matrix $A$, the student said "I'm just rewriting it a different way to try to think about it," as he wrote the matrix equation $A x=b$ and the corresponding vector equation. In that work, Wawro posited that the student's flexibility with the symbolic representations and interpretations of the two equations afforded him ways to successfully reason about and make logical connections between not only linear algebra concepts but also their various symbolic representations. Although they did not use MRC in framing their work, both of these papers present findings consistent with the importance of representational fluency in students' mathematical development in linear algebra. We only found one study in linear algebra that explicitly attended to MRC; Çağlayan [27] had students explore eigenvalues and eigenvectors in a dynamic software program and argued that this provided opportunities for students to invent and develop, critique, and compare the adequacy of various representations, which are key aspects of MRC.
In physics education research, studies about students' understanding in quantum mechanics have examined how students make sense of and use Dirac notation, which is most likely novel to them when they begin a quantum mechanics course. Singh and Marshman [28] showed that even after graduate level instruction in quantum mechanics, students still struggle with Dirac notation, showing inconsistencies in its use among contexts and problems. More closely related with this current study, Gire and Price [29] created four categories of structural features of notation systems and delineated the features for three notations used in quantum mechanics (Dirac, matrix, and wave function). The features identified by the authors are as follows: (a) individuation, or "the degree to which important features are represented as separate and elemental" (p. 5); (b) externalization, or "the degree to which elements and features are externalized with markings included in the representation" (p. 7); (c) compactness; and (d) symbolic support for computation. Using problem-solving interviews with students as insight into how their reasoning interacts with these features, Gire and Price found that students in their study readily used Dirac notation, and that the structural features vary across the different notations as well as among several contexts within quantum mechanics. Building from this work, Schermerhorn et al. [30] investigated students' selection of different representation methods for calculating expectation value problems in quantum mechanics, specifically with expectation values of spin-1/2 particles, energy, and position. Schermerhorn et al. adapted Gire and Price's structural features framework to highlight the computational features present in students' work. Most relevant to the current study, the authors identify computational confidence as an indicator of a student's preference for summation, matrix, or integration notation. The evidence presented supports the claim that students have preferences for notation; in this paper, we hope our use of the MRC framework and our accompanying coding scheme helps tease apart some of these preferences to a finer degree.

\section{METHODS}

Participants for this study were junior physics majors at a large, public, research-intensive university in the Pacific Northwestern United States. They were drawn on a volunteer basis from a class of 35 students in a Paradigms: Spin and Quantum Measurements course. This was a nonstandard course in that it was intense and highly focused on eigentheory in the context of spin systems in quantum mechanics. This course met for 7 class hours per week for three weeks during winter term, and it involved many student-centered activities and discussions. The course used Quantum Mechanics: A Paradigms Approach [31] as its textbook, which presents a spin-first approach to the course. In addition to videotaping each class session, we conducted individual, semi-structured interviews [32] with 8 to 13 


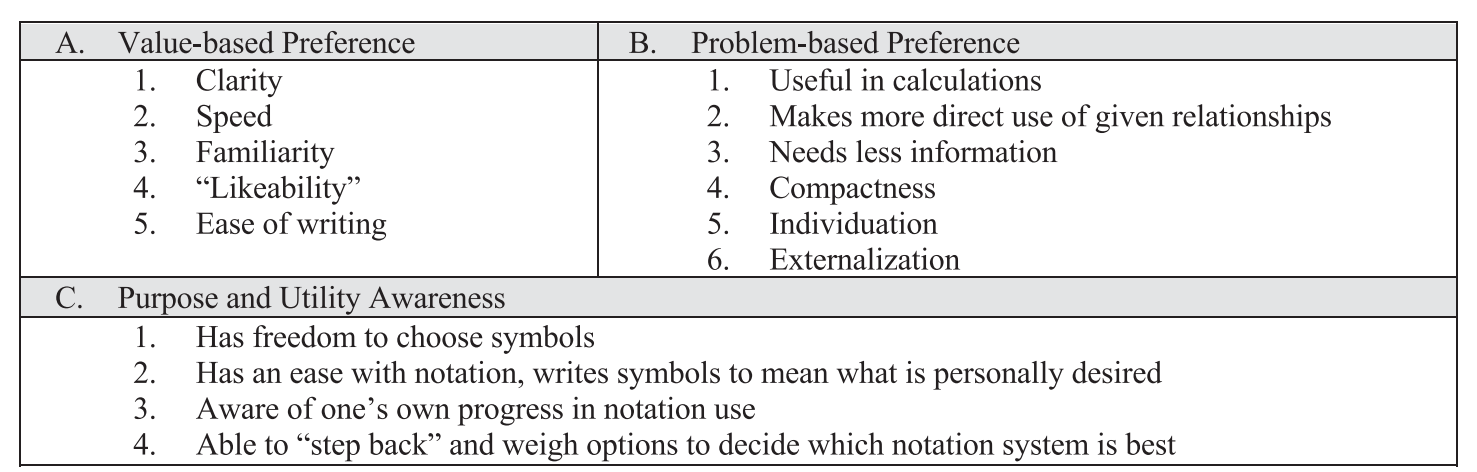

FIG. 1. The list of MRC categories and codes that resulted from our data analysis.

students at the beginning and end of the course. This particular report draws on data gathered during the endof-course interview, in which 8 students participated. The goals of the interview questions were to learn how students reasoned about linear algebra concepts (particularly normalization, basis, and eigentheory), how they reasoned with these concepts as they discussed quantum mechanics concepts and solved quantum mechanics problems, and how they symbolized their work. The results presented here grow from a coding scheme developed to identify MRC as students discuss, describe, and use matrix and Dirac notation to solve quantum mechanics problems.

To begin our analysis, we viewed the videos and observed how students navigated the interview problems, while we kept in mind the overarching research questions regarding students' reasoning about and symbolizing eigentheory in quantum mechanics. Throughout our viewing, we noticed some students were rather fluent in how they talked about and worked with both matrix and Dirac notations, such as the aforementioned Buzz; this fluidity was especially evident in his solution of an expectation value problem. Because this problem seemed to be conducive to eliciting students' understanding, critiques, and comparisons of both notations, we identified which of the eight students had engaged in the expectation value problem in their interview, resulting in a pool of four students to study. The second author then read the transcript or watched the video of the entire interview for all four students, indicating instances in which the student explicitly talked about and reflected on either or both notations. These instances were then collated into a single document with all student identifiers removed. Using this document, each author individually coded for instances of students making explicit metacommentary on the representations they chose to use, specifically attending to ideas related to MRC $[4,33]$. All authors then met collectively to decide on a final set of codes for each transcript section. After the codes were finalized, we used axial coding [34] to place our collective codes into categories of features and characteristics of Dirac and matrix notations to which students seemed to be attuned (Fig. 1).
In Sec. IV, we illustrate our findings by focusing on three students: Milan, Irvin, and Buzz. Milan and Irvin were both double majors in mathematics and physics who had completed one 10-week course in linear algebra and were concurrently enrolled in a second linear algebra course, and Buzz was a double major in physics and nuclear engineering who had completed two 10-week courses in linear algebra. During the interview, Milan and Buzz demonstrated flexibility in reasoning about the concepts we were probing, were articulate in expressing their thinking, and a great deal of MRC was visible and analyzable through their explanations. In order to capture the full extent of their thinking, the interview data is presented in units that are longer than typically presented; however, these sections are then discussed in significant detail.

\section{RESULTS}

Our coding of the data produced three main categories of codes: (A) value-based preference, (B) problem-based preference, and $(\mathrm{C})$ purpose and utility awareness. Each category is composed of 5,6 , and 4 subcodes, respectively (see Fig. 1). We note that our MRC codes have overlap with some aspects of the structural features defined by Ref. [29] and symbol sense defined by [20-21], and students' articulation of some of these ideas fit well within diSessa's framework of MRC.

Categories (A) and (B) are both delineations of one of the aforementioned elements of MRC listed in [4]: "critique and compare the adequacy of representations and judge their suitability for various tasks" (p. 293). Category (A) comprises MRC statements related to preferences students expressed related to some overarching value; statements were similar to sentiments such as one notation being preferred because it is faster to write than another (labeled as A2), more familiar to work with (A3), or easier to write (A5). Other sentiments conveyed a preference based on a particular notation being clearer to read and interpret (A1) or simply because a student "just likes it" (A4).

The MRC codes in category (B) comprise preferential statements students made that seemed more intrinsically 
tied to particular problems or contexts. For example, statements coded with (B2) expressed sentiments consistent with one notation being preferred because it makes more direct use of relationships given in the problem statement (e.g., "sometimes the ket notation is nicer...if you already know the eigenvalues and you just are multiplying by operators"). We note that aspects of this code as well as (B1) (the notation is useful in calculations) are consistent with the structural feature of symbolic support for calculation [29]. In addition, we intentionally use the authors' other three structural features - compactness, individuation, and externalization-as our codes for (B4), (B5), and (B6), respectively, because we saw evidence of them in our data (descriptions of the categorizations are in Sec. II B). A statement was interpreted as consistent with compactness (B4) when it referred to the overall size of a particular notation, e.g., that there are fewer things to write down; we see this as distinct from statements referring to a particular notation being used because it needed less information, coded as (B3). Needing less information within a particular notation may imply that a notation is also seen as "compact," however in our coding we aim not to overinterpret what students might have meant in these statements. We do note that the main category (A, B, or C) that these codes best belong in could be open for discussion. Moreover, some value-based preference codes (such as clarity, A1) could be indicative of students' awareness of notational affordances and constraints (category B), but evidence was lacking for why the student thought a given notation was clearer, more likeable, or easier to write.

Finally, category $(C)$ encapsulates statements that indicate an awareness of representations' purposes and utility. Category (C) captures the other aforementioned element of MRC listed in [3]: "understand the purposes of representations generally... and understand how representations do the work they do for us" (p. 293). We note that these codes in particular are compatible with the aforementioned characteristics of symbol sense [20-21]. Again, student statements consistent with all four (C) codes existed in our data and gave rise to including them in our coding scheme (see Fig. 1). Specifically, statements in which students explicitly comment on their freedom to choose any symbol they want to use in a particular context were captured by code (C1). We coded sentiments in which students described an ease with making a particular notation mean what they wanted it to mean as belonging to category (C2). When this was assigned, the students demonstrated strong agency to choose (and edit if needed) symbols consistent with what they wanted to communicate. Finally, code (C3) encapsulates sentiments of explicit student reflection on their personal progress in using a particular notation, and code (C4) captures when students seemed to "step back" from the work they were doing to consider which notation might be best for a given problem.
Consider the state $|\psi\rangle=-\frac{4}{5}|+\rangle_{x}+i \frac{3}{5}|-\rangle_{x}$ in a spin- $-1 / 2$ system. Calculate the expectation value for the measurement of $S_{x}$.

FIG. 2. The expectation value problem from the interview.

We organize the remainder of our results section according to excerpts from Buzz, Milan, and Irvin, focusing on aspects of their responses that particularly point to and illustrate their MRC (or potential lack thereof, in the case of Irvin). Although not all codes are visible as we present analysis within these three examples, evidence of their existence is found in our remaining interview data.

\section{A. Buzz}

In the beginning of his interview, during his response about how using eigenvectors and eigenvalues in his quantum spins course compared to his experiences using them in other courses, Buzz volunteered that he sometimes explicitly chose between doing calculations in matrix notation or in Dirac notation (see Sec. I). From the transcript, we see that Buzz was aware that there exist multiple legitimate ways to solve the problem, seemingly understanding the various mathematical nuances and implications of his notational choices. His brief explanation highlights sentiments that are consistent with symbol sense characteristics [20-21], such as being "friendly" with symbols and using them flexibly. We add, however, a metacognitive aspect of symbol sense here, noting that Buzz was engaged in self-reflection rather than a researcher analyzing Buzz's engagement with symbols. Thus, we code this as (C4): "able to 'step back' and weigh options to decide which notation system is best." We also note that Buzz was aware of his own progress, commenting that it was "first kind of jarring...to try to do math in kets," but that now it is "kind of easier." We code this as (C3): "aware of one's own progress in notation use."

Because Buzz volunteered expectation value problems as a situation in which he could use either notation, the interviewer had him work on such a problem right away, even though it was prepared to be at the end of the interview. The problem is given in Fig. 2.

Buzz immediately worked on the problem within Dirac notation, saying, "basically to find the expectation value... it's like denoted that way [writes $\langle A\rangle$ ] but really what you're doing is you're taking the, the bra of the state, and then you're putting the operator [writes $=\langle\psi|A| \psi\rangle]$ in the middle of the inner product" [Fig. 3(a) line 1]. He continued,

Buzz: Because the A here is $S_{x}$ and then this is uh, this is expressed entirely in the $x$ basis kets, you know that $S_{x}$ is just going to um, like apply its eig-eigenvalues to these, so, so like the eigenvalue co-uh, corresponding to plus $x$ 


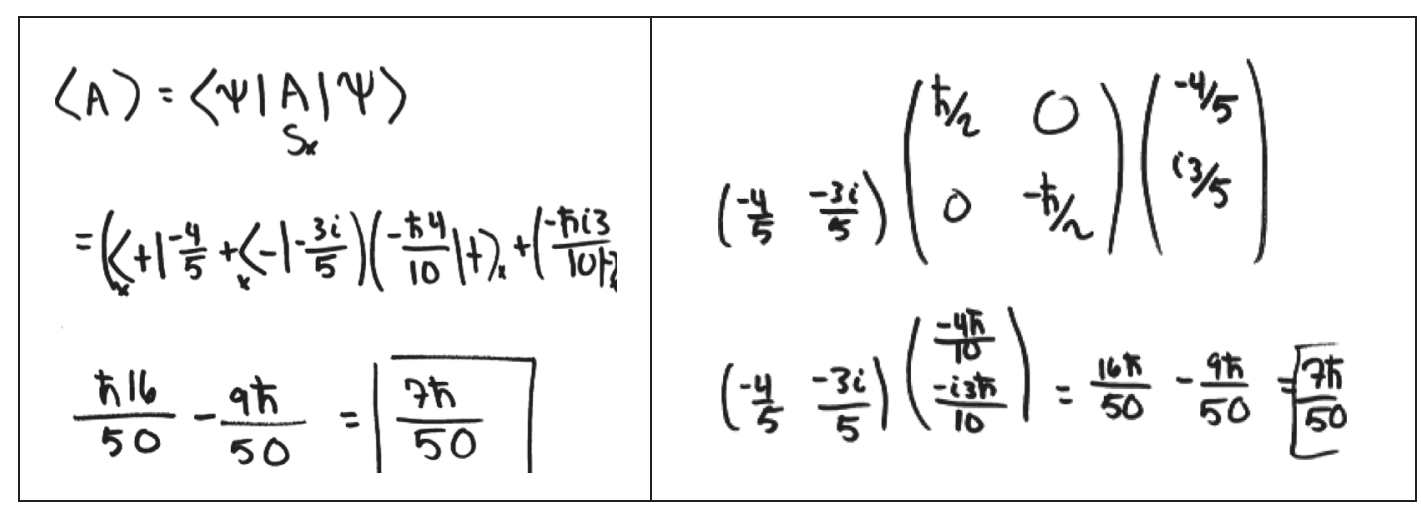

(a)

(b)

FIG. 3. Buzz's expectation value problem work, in Dirac notation (a) and matrix notation (b).

is going to be $+\hbar / 2$ and the, the eigenvalue corresponding to minus $x$ is going to be $-\hbar / 2$, so you end up with this equation that looks like this. You have the bras on one side, um, which is $x$ basis, negative 4 over 5 , uh plus, the minus $x$ basis, and then that changes sign because you're taking the Hermitian adjoint, $3 i$ over 5 , [see Fig. 3(a) line 2]. And then now you can just take this but just apply the, the, the eigenvalues to each one, um, so you have, uh, $-4 \hbar / 10$ which will cancel out but, uh and then plus $x$ ket, and then plus, uh, mi- that's gonna be $a-\hbar 3 i / 10$, and then put a little minus ket there [Fig. 3(a), line 2]. And then, and then you just, you're looking at the, the products of each of these things, so this times this gives you, uh, $\hbar 16 / 50$, and then you're adding it to this, which you have a negative and negative, so that's positive, but then you have $i^{2}$ so it's minus $9 \hbar / 50$ again. So your expectation value ends up

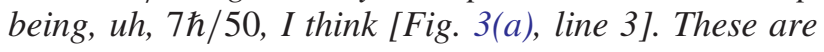
still kind of new problems, but that's, that's would be my answer at this point.

Note that his work in Fig. 3(a) involved the state's expansion and use of eigenvector equations for $S_{x}$ in ket notation. We point out his speed and ease in calculating $S_{x}|\psi\rangle$, which he did by knowing "that $S_{x}$ is just going to um, like apply its eig- eigenvalues to these" components of the psi state vector. He also noted that expectation value problems were "kind of new." We add that Dirac notation itself was first introduced to the students during this course; as such, Buzz was clearly quick to use and understand this representation (an additional quality of MRC [5]).

After discussing what his solution meant physically, the interviewer asked: "Before you were talking about bra-ket versus matrix notation, you brought up an expectation value as an example of like, either or both, so can you, now that you had this problem, kind of revisit that?" Buzz immediately solved the problem completely within matrix notation [Fig. 3(b)], explaining his steps as he progressed:
Buzz: So, if we're strictly in the, in the, the plus and minus ket bas-or $x$ basis, then you can write this vector as a, as a, like that ket as a, as -4 over 5, and uh i3 over 5, [writes column vector, Fig. 3(b), line 1] and then the, and then the bra would then be, um, -4 over 5 and then minus, minus $3 i$ or, sorry-3i over 5 [writes row vector, Fig. 3(b), line 1] ... And then you have the operator in the middle...the operator in this case is $S_{x}$, and we're in the $x$ basis so it's just becomes $\hbar / 2,0,0$, minus $\hbar / 2$, [writes $2 \times 2$ matrix, Fig. 3(b), line 1]. And you can do it this way, you can-you can do it-bring this over here and then do that [indicates multiplying the matrix times the ket column vector] and then, you're gonna get a vector and you can dot that vector with that one [indicates the row vector]. But that's, that- that-you can do it that way but, I think this [pointing to his work shown in Fig. 3(a)] is easier.

Not being sure what he meant by "easier," which does indicate some aspect of MRC, the interviewer asked him to go ahead and finish the problem using matrix notation. He calculated the matrix times the column vector and that result times the row vector, determining $7 \hbar / 50$ easily and quickly [Fig. 3(b), line 2], stating, "which is the same as we got before, thankfully." The interviewer then asked Buzz to reflect on his preference between the two notations:

Buzz: Uh...To be honest, I don't really, I don't really know why I prefer this [Fig. 3], I think it's just because, um, I like this notation. This- this specific notation [Fig. 3(a), line 1] like this to me is like a cleaner way of writing that [Fig. 3(b), line 1] because that- I mean this and that [touching lines 1 in both figures] Ifeel like are your starting points, so you, you start here with this nice, like, looking thing [traces his finger under $\langle\psi|A| \psi\rangle]$, or you start here with this big array of numbers [puts open hands around Fig. 3(b)], and I prefer this [Fig. 3(a), line 1], even though you have to expand this into basically the same amount of information [Fig. 3(a), line 2]. And also, the nice thing about, about this [Fig. 3(a), line 1], 


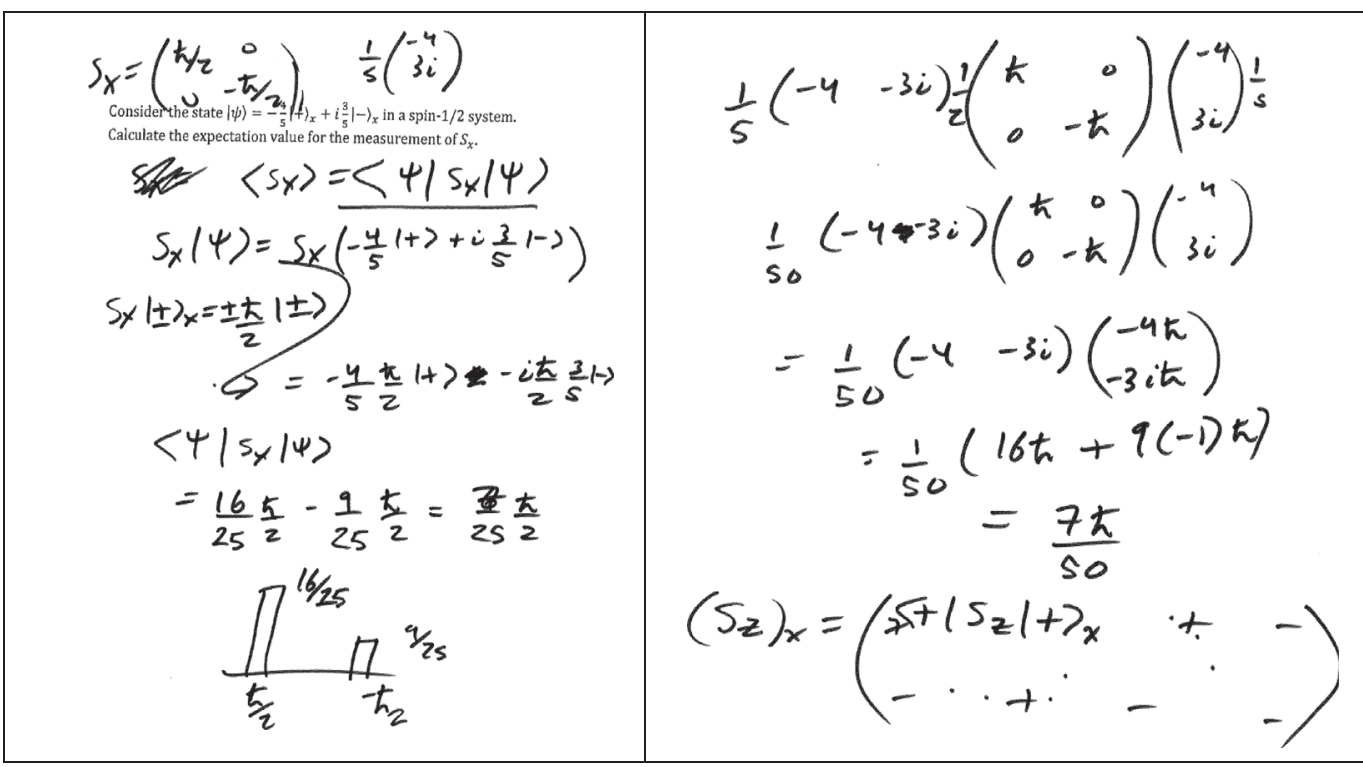

(a)

(b)

FIG. 4. Milan's work on the expectation value problem, in Dirac notation (a) and matrix notation (b).

is it-actually this is really why it's better-is because you can, you can say ok $S_{x}$ works- acts directly on these kets, you can just get rid of the matrix altogether. Whereas this one [Fig. 3(b)] is like, you have to recognize that, that, that you're in this basis and it's just, yeah it's not as, it's not as nice um, 'cause basically when you do this matrix multiplication, you have to do this matrix multiplication just to get where I got from here to here [Fig. 3(a), line 1 to line 2], which was literally I just said ok, looked at this and then I expanded it out because I knew that this $\left[S_{x}\right]$ acting on that [first term of $|\psi\rangle]$ was gonna be $\hbar / 2$ and this [ $S_{x}$ ] acting on that [second term of $|\psi\rangle$ ] was going to be $-\hbar / 2$, so I just put them over there, whereas here kinda have to do that vector matrix thing first before you get those values.

This excerpt begins with two value statements: that Buzz prefers Dirac notation because he likes it (A4: likeability) and because it provides a cleaner way of writing the desired information (A1: clarity). We see his use of "nice looking thing" and "big array of numbers" in comparison to one another as an example of compactness (B4). He also compares line 1 in 2(a) and line 2 in 2(b) regarding the "amount" of information they convey, which involves reflection on the physical and mathematical content expressed in the compared notations (B6: externalization). Finally, acting directly on the expansion in terms of the eigenstates of the operator allowed him to forego the matrix calculation entirely, which speaks to Buzz's view of the utility of a notation for calculation in a specific problem (B1) by making direct use of given relationships (B2).

When asked about his notation preferences if the basis expansion of a given state vector and the operator "didn't match," (here they were both in the $x$ basis), Buzz recalled a problem from their last homework that was "actually easier...to do the matrix multiplication," stating "you don't want to have to change these kets into different bases all over the place 'cause they're already all written in the same basis and you know what the operator is in that basis so you might as well just, do the matrix multiplication." This speaks to his awareness of using given relationships directly (B2) as well as being able to choose which notation system is best for a certain situation (C4). Finally, when asked if the notions of basis or eigenvectors and eigenvalues come up more in one notation than the other, Buzz stated, "certainly...every time you write down a ket you're, you're very conscious of what basis you're in. In this one [points to Fig. 3(b)] it's just kind of implied...all this [is] in the same basis, so you're just, you're just writing out numbers and arrays of numbers, but here [in Fig. 3(a)] I think that you're definitely more aware of what basis you're in when you're using this, because you have to be." This explanation is consistent with Gire and Price's [29] notion of externalization (B6) in that the ket notation allowed problem features, namely, basis, to be externalized for Buzz in a way that matrix notation did not.

\section{B. Milan}

We focus our examples of Milan's MRC on his work with the expectation value problem. Like Buzz, he first completed the problem in Dirac notation [see Fig. 4(a)]:

Milan: So, this is very convenient because it's in the $S_{x}$ basis. Um, so, basically all we need to do is put $S_{x}$ in some matrix representation as-well, do we need to do 
matrix representation? I don't think we do. So, let's not worry about that. So, the expectation value of $S_{x}$ is defined as this $\psi$, and the $S_{x}$, and $\psi$ [writes $\left.\left\langle\psi\left|S_{x}\right| \psi\right\rangle\right]$. And we're just going to include the $\psi$ 's in this-We're just going to drop the $x$ subscript and just assume that it's, um, the $x$ basis....

As Milan began his work on this problem, he thought aloud about his approach, first stating he would put $S_{x}$ in a matrix form but then deciding he did not need to do so. This speaks to Milan's ability to "step back" and weigh options to decide which notation system is best (C4). Once he decided to solve the problem within Dirac notation, he explained his choice to "drop the $x$ subscript" from the expansion of the state $\psi$ in terms of the $S_{x}$ eigenbasis (note the missing $x$ subscripts on the $| \pm\rangle$ kets in Fig. 4(a) demonstrating Milan's ease with notation in writing and engineering symbols to mean what he personally desired (C2). After working through the calculations, during which Milan correctly explained his steps to solve the expectation value problem [Fig. 4(a)], the interviewer asked him to comment more on his rather explicit choice not to use matrix notation:

Int: When you first started, you almost started using matrix notation, and then you decided not to. Can you talk more about that?

Milan: So, ket-because you know this eigenvalue equation, ket notation just skips all that. Um, if you wanted to, you could have written this out as, uh, $\frac{1}{5},-4$, -3i [writes row vector in Fig. 4(b)]. But, you can see that's more confusing to go through-and then you have to look at $S_{x}$ and, where you can write it as $\hbar / 2,-\hbar / 2$, 0 , 0 , and you have to do matrix multiplication. Um, actually, that might be quicker, honestly...

Milan's statement, "Because you know this eigenvalue equation, ket notation just skips all that" demonstrates his belief that Dirac notation makes a more direct use of relationships (B2), specifically those contained in the eigenvalue equations $S_{x}| \pm\rangle_{x}= \pm \hbar / 2| \pm\rangle_{x}$. He also stated that one could have written the given $\psi$ as a column vector [another example of his awareness of choice in notation system (C4)], but writing the states as column vectors would be more confusing in this situation; this evidences Milan's belief that Dirac notation can have a greater level of clarity than matrix notation (A1).

Next, Milan summarized the matrix symbols and operations that would be necessary to complete the calculation, which led him to reflect aloud that matrix notation might actually be faster for him in this problem; this shows a preference for matrix notation based on a value judgment of speed (A2). The interviewer prompted Milan to calculate the expectation value using matrix notation [see Fig. 4(b)], through which he arrived at the same answer he obtained through his calculations in Dirac notation.
Milan: Which is, the exact same answer we got before, and it was substantially quicker. And in-I mean, I guess it depends how good you were with this kind of notation [pointing to his work in Fig. 4(a)]. 'Cause, this [Dirac notation] is, I learned two and a half weeks ago, and this [pointing to work in Fig. 4(b)] I learned almost a year ago.

In this final quote, Milan spontaneously reflected more about the two notation options, noting aspects of familiarity and speed in using matrix notation (A2, A3) as reasons someone might choose it over Dirac notation. Additionally, Milan demonstrated an awareness of his own progress in understanding Dirac notation, noting it takes time to progress in using and understanding new notational systems (C3).

\section{Irvin}

To further explore the possible interdependence of conceptual understanding and metarepresentational competence, we examine a third student, Irvin, whose interaction with the expectation value problem (Fig. 2) did not have as smooth of a start as Milan and Buzz. None of Irvin's actions or words during his interaction with the problem (or his reflection on it) were able to be coded with our MRC framing. We cannot claim that Irvin did not possess any MRC; rather, all we can know is that an MRC way of thinking did not seem activated for Irvin at that time because we did not see evidence of MRC as he discussed the problem and his solution. Let us consider his interaction. As soon as he finished reading the problem, he stated the values $16 / 25$ and $9 / 25$ as he wrote them, stating "Now this one I'm sure of." When asked to explain, he said "because it's the same thing as if this was, so, if I wanted I can make a similar problem, I can be like, "oh, you got psi, and it's- and it's equal to negative 4 over 5 plus, eh, plus $i 3$ over 5 minus, uh what's- it's in the $z$ basis? I mean done, that's the same thing." His solution to both the original and his invented problem would be correct if he were asked to calculate the probabilities of the measurements of spin up and down along the $y$ axis and $z$ axis, respectively, which involved making sure the basis representation of psi matched the axis of orientation for the measuring apparatus.

The interviewer then followed up by asking "tell me about the difference in the wording, "cause that one [the current problem] talks about expectation value versus a probability of up or down [points to the previous interview problem]," to which Irvin seemed to express surprise with the exclamation, "wait, what?" He continued with the explanation:

Irvin: Oh, alright. Yeah, see I got, on the homework problems, they- they, they got me on this one, they gotthey docked points, I did not put myself an $h$ bar over 2 


\section{$\frac{16}{25} \frac{5}{2} \quad \frac{9}{25} \frac{-5}{2}$}

FIG. 5. Irvin's second solution to the expectation value problem.

here and I put not- put negative h bar over 2 here [writes those next to the values $16 / 25$ and 9/25, see Fig. 5]. Because it's an expectation value, so, technically if I was- if I was gonna do this, like, with perfect, like, wording, based on the expectation value words there, then yeah, I would've, I would've put the h bar, uh, I woulda-I woulda done everything right [underlines the two terms in Fig. 5]. But, I- I usually- I- I see probabilities and expectation val-oo, sorry, expectation value? [again, seems a bit surprised] I'm sorry, I thought you meant, never mind. Expectation value's th, this thing. [writes $\left\langle S_{x}\right\rangle$ ]

We note that this exchange occurred because the interviewer pursued Irvin's original answer, not because Irvin noticed it was incorrect. Irvin's comments of "they got me" and "they docked points" do not convey that Irvin considered his answer incorrect conceptually; rather, they seem to convey that he thought he just didn't write the symbols the homework grader and/or professor wanted to see. This leads us to hypothesize that he may not have had a richly developed connection of meaning behind the symbols and the physical and/or mathematical interpretation of them. An alternative explanation is that Irvin's MRC capabilities may not have been evident because his content confusion (state probabilities versus expectation value) may have dominated his thinking, leaving little space to make visible any of his considerations relevant to demonstrating MRC.

In this case, the symbols in question go beyond just "vector notation versus Dirac notation" to include $\hbar / 2$ and how to write it sensibly (for instance, the symbolic concatenation in the second term formally conveys subtraction rather than multiplication). His continued explanation of if he were to do "this with perfect wording" and do "everything right" included writing the correct signed versions of $\hbar / 2$ next to the probability terms, but he did not carry out the summing calculation across the two terms. Finally, after he seemed to have some insightful moment, he "defined" expectation value by writing the symbols " $\left\langle S_{x}\right\rangle$ " without saying any words or explanation with it, so we cannot be sure what " $\left\langle S_{x}\right\rangle$ " meant to him.

Because Irvin seemed to change his way of thinking during his response, the interviewer asked him, "What just happened?" Irvin continued to explain:

Irvin: I thought when you said expectation value you meant like the, the probabilities with their eigenvalues associated with them. If, if I was gonna, I'd add these

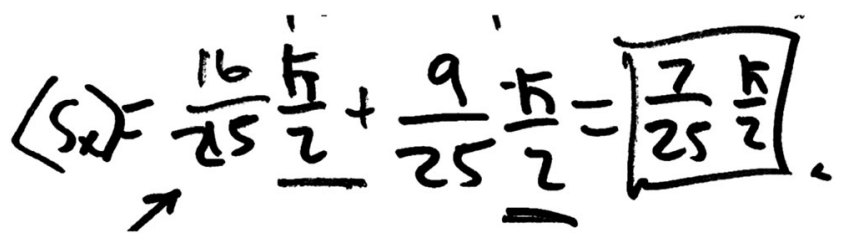

FIG. 6. Irvin's final solution to the expectation value problem.

guys up [writes a plus sign between the two terms from Fig. 5] and I'd get something like, this would be the $S_{x}$ [writes " $\left\langle S_{x}\right\rangle=$ " in front of his new sum]. Or would it be? Wait, [reading the problem again] "of $S_{x}$," yes, ok yeah, so then this would be, eh, just... 16 minus 9 [writing] $h$ bar over 2. So that would be the expectation value [puts a box around $\frac{7}{25} \hbar / 2$, see Fig. 6]. This is the average, what you'd expect in experiment.

Finally, the interviewer agreed with Irvin's answer and asked him to explain everything again, to which he replied

Irvin: I was confused, when they said expectation value, I wasn't sure if they meant the probabilities just multiplied by the eigenvalue it's associated with. 'Cause, on one of the problems on the homework, I got docked points 'cause I just put a probability and they're like [switches voice to impersonate an unknown homework grader] "Well you didn't, you didn't have the $h$ bar."

We call attention to a number of aspects of Irvin's response. First, we note that Irvin defined expectation value as "the probabilities with their eigenvalues associated with them." In this wording (and in a subsequent, similar wording), he did not mention adding the two resulting terms, and, in fact, his response in Fig. 5 did not include the summation. We interpret this conceptualization as if Irvin was blending the probabilities of measuring the two possible outcomes with the expectation (mean) value of the measurement over a large number of experiments. Next, we note that Irvin does eventually correctly carry out the needed procedure of adding terms to result in one value for expectation value for $S_{x}$ (see Fig. 6). He also associated the correct symbols $\left\langle S_{x}\right\rangle$ and an accurate physical interpretation of "This is the average, what you'd expect in experiment" with his solution.

Lastly, we note that in Irvin's final explanation he returns to discussing "the probabilities just multiplied by the eigenvalue it's associated with" as expectation value. He also mentioned again one of his homework answers not being given full marks: "I got docked points 'cause I just put a probability and they're like 'you didn't have an $\mathrm{h}$ bar'." It is not clear whether he realized the conceptual distinction between probability and expectation value if, in his judgment, all he did was neglect to include a certain symbol. The two different types of questions have two 
different forms of answers (e.g., probabilities are real values between 0 and 1 inclusively) because of what they are conceptually. One explanation for this could be that deep conceptual understanding of the quantum mechanical concepts at play involves coordination with what the associated symbols mean and why they are important. Although we do not see evidence of Irvin demonstrating strong MRC at this moment, it is possible that Irvin would do so in other scenarios and with other problems and prompts. In the data presented, it is possible that Irvin was so focused on the content and correctly interpreting the problem (which he eventually does) that he simply did not engage in reasoning that demonstrated his MRC.

\section{DISCUSSION}

In this article we shared our analysis of the metarepresentational competence of three students as they engaged in solving a quantum mechanics problem involving linear algebra. We analyzed two students who serve as paradigmatic examples of students' power and flexibility within different notation systems, and analysis of a third student was given as a point of contrast. In addition to adapting MRC as a helpful construct for characterizing student understanding at the intersection of undergraduate mathematics and physics, we demonstrated how students' rich understanding of linear algebra and quantum mechanics includes and is aided by their understanding and flexible use of different notational systems.

We offer three reasons why this work is important to mathematics and physics researchers and educators. First, similar to one of the purposes of Ref. [2], our report acts as an alternative narrative to a number of articles in physics education that focus on students' difficulties or misconceptions in learning quantum mechanics (e.g., Refs. [3537]), and more specifically with the notations used in quantum mechanics $[28,38]$. We have shown students' abilities to critique and understand the general purposes of the various notations used within quantum mechanics, and how their metarepresentational competence can even include some ideas about strengths and weaknesses of the notations that align with expert-determined structural aspects of quantum representations [29]. Our findings for Buzz and Milan are consistent with Smith's [39] characterization of student "observable expertise" as rich, highly adaptable to problem specifics, and composed of a wide variety of strategies. Instead of focusing on the identification of students' difficulties with notation, we highlight what students attend to when using (or choosing to use) a particular notation and how this seems to impact their decision when using a particular notation; this is consistent with recent calls for research from antideficit perspectives with respect to investigating student learning [40]. In turn, educators might examine how they can build upon the metarepresentational competence students already have to help deepen and strengthen their understanding of quantum mechanical and mathematical representations.

Second, most studies on MRC have been about inventing and critiquing representations, but our study extends knowledge about MRC by demonstrating that MRC can be relevant and helpful for understanding and using canonical representations. In particular, we presented codes we created for identifying and analyzing students' MRC in their talk about eigentheory and quantum mechanics, as well as general categories for the types of MRC these codes indicate students demonstrate. This coding scheme still has the potential to be expanded, extended, and refined, especially as students' MRC within quantum mechanics and linear algebra is further explored, and as students' MRC is studied within other mathematical and physical contexts. Our hope is this coding scheme will facilitate analysis of students' understanding of representations within mathematics and physics, as well as help researchers gain insight into the richness of students' overall understanding of mathematical and physical concepts. Furthermore, we hope the identification of particularly useful and powerful elements of MRC, such as those found in analyzing experts or strong students, will help educators know the types of thinking and reasoning to emphasize and cultivate in teaching their students. Education researchers could then search for teaching methods, questions, and curricular materials that would support students in developing their MRC in these productive ways. We echo the suggestion that "in order to optimize physics learning in interactive contexts, physics teachers need to know more about the range of persistent representations available, and their associated disciplinary affordances" (Ref. [19], p. 665). Thus, helping students develop deep understanding of quantum mechanics and linear algebra should include providing opportunities for students to use and improve their metarepresentational competence.

Third, this analysis furthers what is known related to how student understanding of content, in this case, specifically linear algebra and quantum mechanics, includes and is aided by their understanding and flexible use of different notational systems. As mentioned in Ref. [4], one aspect of educational research is to seek ways to help students develop deeper understanding of science and mathematics; an important piece of this deeper understanding includes knowing "not only how to operate scientific apparatus (from strategies that solve problems, to representations and concepts), but also understanding how and why they work, and even being able to generate and judge alternative means" (p. 299). Within that, we particularly highlight the importance of students knowing not only how to "operate" representations (such as the Dirac or matrix notational systems) but also how they work and how to judge alternative representational approaches, as a key piece of a deep understanding of science and mathematics itself. Furthermore, Meira [7] states that "problem-solving 
strategies are not simply applied to representations, but their very emergence may depend on the existence of specific inscriptions already displayed on paper" (p. 101). In other words, a problem-solving approach is itself, rather than being distinct from, intrinsically tied to a notational system; this plays out in our data, most readily in the MRC codes within the "problem-based preference" category. We note that Buzz and Milan, two of the strongest students within our study in regards to their understanding of linear algebra and quantum mechanics concepts, also demonstrated particularly articulate and thoughtful MRC. This may suggest that a deep understanding of the physical and mathematical concepts creates a conducive environment for developing strong MRC, while MRC simultaneously helps students develop a rich understanding of physical and mathematical concepts. As Sherin [41] noted, "We do students a disservice by treating conceptual understanding as separate from the use of mathematical notations" (p. 482).

We conclude by suggesting that MRC could be further explored as aspects of procedural and conceptual understanding [42-43] of advanced mathematics and physics content. Hiebert and Lefevre [42] defined conceptual knowledge as "knowledge that is rich in relationships... a connected web of knowledge, a network in which the linking relationships are as prominent as the discrete pieces of information" (pp. 3-4), and procedural knowledge as "familiarity with the individual symbols of the system and with the syntactic conventions for acceptable configurations of symbols" (p. 7). Star [43] defined deep procedural knowledge as "knowledge of procedures that is associated with comprehension, flexibility, and critical judgment and that is distinct from (but possibly related to) knowledge of concepts" (p. 408). We consider these in light of diSessa and Sherin's [5] description of MRC:
The full range of capabilities that students (and others) have concerning the construction and use of external representations. MRC includes the ability to select, produce and productively use representations but also the abilities to critique and modify representations and even to design completely new representations. (p. 386)

In analyzing the MRC of students as they engaged in solving a quantum mechanics expectation value problem, we were able to document knowledge that is rich in relationships, familiarity with symbols and their syntactic conventions, and flexibility and critical judgment as students chose and carried out procedures - all of which are aspects of procedural and conceptual knowledge.

Future research could continue to investigate the relationship between MRC and deep understanding of advanced mathematics and physics content, as well as the impact of explicitly trying to teach students to develop strong MRC on student understanding and performance.

\section{ACKNOWLEDGMENTS}

This material is based upon work supported by the National Science Foundation under Grant No. DUE1452889. The authors thank Andrea diSessa, Jeffrey Rabin, Michael Wittmann, and Michelle Zandieh for their insights on the data. Any opinions, findings, and conclusions or recommendations expressed in this material are those of the authors and do not necessarily reflect the views of the NSF. We are thankful for Kevin Watson's integral involvement with this research, which included contributions to study design and data collection, theoretical and methodological framing, data analysis, and writing.
[1] National Research Council, Discipline-based education research: Understanding, and improving learning in undergraduate science, and engineering, edited by S. R. Singer, N. R. Nielsen, and H. A. Schweingruber, Committee on the Status, Contributions, and Future Direction of Discipline Based Education Research. Board on Science Education, Division of Behavioral and Social Sciences and Education (The National Academies Press Washington, DC, 2012).

[2] A. diSessa, D. Hammer, B. Sherin, and T. Kolpakowski, Inventing graphing: Meta-representational expertise in children, J. Math. Behav. 10, 117 (1991).

[3] M. Wawro, K. Watson, and W. Christensen, Metarepresentational competence with linear algebra in quantum mechanics, in Proceedings of the $20^{\text {th }}$ Annual Conference on Research in Undergraduate Mathematics Education, San Diego, CA, edited by A. Weinberg,
C. Rasmussen, J. Rabin, M. Wawro, and S. Brown (Special Interest Group of the Mathematics Association of America for Research in Undergraduate Mathematics Education, Washington, DC, 2017), pp. 326-337. http://sigmaa.maa .org/rume/RUME20.pdf.

[4] A. diSessa, Metarepresentation: Native competence and targets for instruction, Cognit. Instr. 22, 293 (2004).

[5] A. diSessa and B. Sherin, Meta-representation: An introduction, J. Math. Behav. 19, 385 (2000).

[6] F. Azevedo, Designing representations of terrain: A study in metarepresentational competence, J. Math. Behav. 19, 443 (2000).

[7] L. R. D. L. Meira, Mathematical representations as systems of notations-in-use, in Symbolizing, Modeling, and Tool Use in Mathematics Education, edited by $\mathrm{K}$. Gravemeijer, R. Lehrer, B. Van Oers, and L. Verschaffel 
(Kluwer Academic Publishers, Dordrecht, Netherlands, 2002), pp. 87-103.

[8] B. Sherin, A. A. diSessa, and D. Hammer, Dynaturtle revisited: Learning physics through collaborative design of a computer model, Interact. Learn. Environ. 3, 91 (1993).

[9] A. Iszák, G. Çağlayan, and J. Olive, Meta-Representation in an Algebra I classroom, J. Learn. Sci. 18, 549 (2009).

[10] A. Iszák, "We want a statement that is always true": Criteria for good algebraic representations and the development of modeling knowledge, J. Res. Math. Educ. 34, 191 (2003).

[11] R. J. Beichner, Testing student interpretation of kinematics graphs, Am. J. Phys. 62, 750 (1994).

[12] P. B. Kohl, D. Rosengrant, and N. D. Finkelstein, Strongly and weakly directed approaches to teaching multiple representation use in physics, Phys. Rev. ST Phys. Educ. Res. 3, 010108 (2007).

[13] D. E. Meltzer, Relation between students' problem-solving performance and representational format. Am. J. Phys. 73, 463 (2005).

[14] D. Rosengrant, A. Van Heuvelen, and E. Etkina, Do students use and understand free-body diagrams?, Phys. Rev. ST Phys. Educ. Res. 5, 010108 (2009).

[15] M. Hill and M.D. Sharma, Students' representational fluency at university: A cross-sectional measure of how multiple representations are used by physics students using the representational fluency survey, Eurasia J. Math. Sci. Technol. Educ. 11, 1633 (2015).

[16] M. Hill, M. D. Sharma, and H. Johnston, How online learning modules can improve the representational fluency and conceptual understanding of university physics students, Eur. J. Phys. 36, 045019 (2015).

[17] G. Taasoobshirazi, M. Bailey, and J. Farley, Physics Metacognition Inventory Part II: Confirmatory factor analysis and Rasch analysis, Int. J. Sci. Educ. 37, 2769 (2015).

[18] G. Taasoobshirazi and J. Farley, Construct validation of the physics metacognition inventory, Int. J. Sci. Educ. 35, 447 (2013).

[19] T. Fredlund, J. Airey, and C. Linder, Exploring the role of physics representations: an illustrative example from students sharing knowledge about refraction, Eur. J. Phys. 33, 657 (2012).

[20] A. Arcavi, Symbol sense: Informal sense-making in formal mathematics, For the Learning Math. 14, 24 (1994).

[21] A. Arcavi, Developing and using symbol sense in mathematics, For the Learning Math. 25, 42 (2005).

[22] G. Harel and J. Kaput, The role of conceptual entities and their symbols in building advanced mathematical concepts, in Advanced Mathematical Thinking, edited by D. Tall (Kluwer Academic Publishers New York, NY, 2002).

[23] J. Novotná and M. Hoch, How structure sense for algebraic expressions or equations is related to structure sense for abstract algebra, Math. Educ. Res. J. 20, 93 (2008).

[24] D. O. Tall, Building theories: The three worlds of mathematics, For the Learning Math. 24, 29 (2004).

[25] J. Hillel, Modes of description and the problem of representation in linear algebra, in On the Teaching of
Linear Algebra, edited by J.-L. Dorier (Kluwer, Dordrecht, Netherlands, 2000), pp. 191-207.

[26] M. Wawro, Reasoning about solutions in linear algebra: The case of Abraham and the Invertible Matrix Theorem, Int. J. Res. Undergraduate Math. Educ. 1, 315 (2015).

[27] G. Çağlayan, Making sense of eigenvalueeigenvector relationships: Math majors' linear algebra-geometry connections in a dynamic environment, J. Math. Behav. 40, 131 (2015).

[28] C. Singh and E. Marshman, Investigating student difficulties with Dirac notation, in Proceedings of the 2013 Physics Education Research Conference, Portland, OR, edited by P. V. Engelhardt, A. D. Churukian, and D. L. Jones (AIP, New York, 2013).

[29] E. Gire and E. Price, Structural features of algebraic quantum notations, Phys. Rev. ST Phys. Educ. Res. 11, 020109 (2015).

[30] B. P. Schermerhorn, G. Passante, H. Sadaghiani, and S. J. Pollock, Exploring student preferences when calculating expectation values using a computational features framework, Phys. Rev. Phys. Educ. Res. 15, 020144 (2019).

[31] D. McIntyre, C. A. Manogue, and J. Tate, Quantum Mechanics: A Paradigms Approach, 1st ed. (AddisonWesley, Boston, MA, 2012).

[32] R. H. Bernard, Research Methods in Cultural Anthropology (Sage Publications Newbury Park, CA, 1988).

[33] A. diSessa, Students' criteria for representational adequacy, in Symbolizing, Modeling and Tool Use in Mathematics Education, edited by K. Gravemeijer, R. Lehrer, B. Van Oers, and L. Verschaffel (Kluwer Academic Publishers Dordrecht, Netherlands, 2002), pp. 105-129.

[34] B. Glaser and A. Strauss, The Discovery of Grounded Theory: Strategies for Qualitative Research (Aldine Publishing Company Chicago, IL, 1967).

[35] I. D. Johnston, K. Crawford, and P. R. Fletcher, Student difficulties in learning quantum mechanics, Int. J. Sci. Educ. 20, 427 (1998).

[36] C. Singh, Student understanding of quantum mechanics. Am. J. Phys. 69, 885 (2001).

[37] D. F. Styer, Common misconceptions regarding quantum mechanics. Am. J. Phys. 64, 31 (1996).

[38] C. Singh and E. Marshman, A review of student difficulties in upper-level quantum mechanics, Phys. Rev. ST Phys. Educ. Res. 11, 020117 (2015).

[39] J. P. Smith, Competent reasoning with rational numbers, Cognit. Instr. 13, 3 (1995).

[40] A. P. Adiredja, Anti-deficit narratives: Engaging the politics of research on mathematical sense making, J. Res. Math. Educ. 50, 401 (2019).

[41] B. Sherin, A comparison of programming languages and algebraic notation as expressive languages for physics, Int. J. Comput. Math. Learning 6, 1 (2001).

[42] J. Hiebert and P. Lefevre, Conceptual and procedural knowledge in mathematics: An introductory analysis in Conceptual and Procedural Knowledge: The Case of Mathematics, edited by J. Hiebert (Lawrence Erlbaum Associates Hillsdale, NJ, 1986).

[43] J. R. Star, Reconceptualizing procedural knowledge, J. Res. Math. Educ. 36, 404 (2005). 